\title{
An Erdös-Révész type law of the iterated logarithm for reflected fractional Brownian motion
}

\author{
K. Dębicki ${ }^{1}$ K. M. Kosiński ${ }^{1}$
}

Received: 5 January 2017 / Revised: 28 April 2017 / Accepted: 2 May 2017/

Published online: 18 May 2017

(C) The Author(s) 2017. This article is an open access publication

\begin{abstract}
Let $B_{H}=\left\{B_{H}(t): t \in \mathbb{R}\right\}$ be a fractional Brownian motion with Hurst parameter $H \in(0,1)$. For the stationary storage process $Q_{B_{H}}(t)=$ $\sup _{-\infty<s \leq t}\left(B_{H}(t)-B_{H}(s)-(t-s)\right), t \geq 0$, we provide a tractable criterion for assessing whether, for any positive, non-decreasing function $f$, $\mathbb{P}\left(Q_{B_{H}}(t)>f(t)\right.$ i.o. $)$ equals 0 or 1 . Using this criterion we find that, for a family of functions $f_{p}(t)$, such that $z_{p}(t)=\mathbb{P}\left(\sup _{s \in\left[0, f_{p}(t)\right]} Q_{B_{H}}(s)>f_{p}(t)\right) / f_{p}(t)=$ $\mathscr{C}\left(t \log ^{1-p} t\right)^{-1}$, for some $\mathscr{C}>0, \mathbb{P}\left(Q_{B_{H}}(t)>f_{p}(t)\right.$ i.o. $)=1_{\{p \geq 0\}}$. Consequently, with $\xi_{p}(t)=\sup \left\{s: 0 \leq s \leq t, Q_{B_{H}}(s) \geq f_{p}(s)\right\}$, for $p \geq 0, \lim _{t \rightarrow \infty} \xi_{p}(t)=\infty$ and $\lim \sup _{t \rightarrow \infty}\left(\xi_{p}(t)-t\right)=0$ a.s. Complementary, we prove an Erdös-Révész type law of the iterated logarithm lower bound on $\xi_{p}(t)$, i.e., $\lim \inf _{t \rightarrow \infty}\left(\xi_{p}(t)-\right.$ $t) / h_{p}(t)=-1$ a.s., $p>1$; $\liminf _{t \rightarrow \infty} \log \left(\xi_{p}(t) / t\right) /\left(h_{p}(t) / t\right)=-1$ a.s., $p \in$ $(0,1]$, where $h_{p}(t)=\left(1 / z_{p}(t)\right) p \log \log t$.
\end{abstract}

Keywords Extremes of Gaussian fields · Storage processes · Fractional Brownian motion $\cdot$ Law of the iterated logarithm

AMS 2000 Subject Classifications Primary: 60F15 - 60G70; Secondary: 60G22

K. M. Kosiński

Kamil.Kosinski@math.uni.wroc.pl

K. Dębicki

Krzysztof.Debicki@math.uni.wroc.pl

1 Mathematical Institute, University of Wrocław, pl. Grunwaldzki 2/4, 50-384 Wrocław, Poland 


\section{Introduction and main results}

The analysis of properties of reflected stochastic processes, being developed in the context of classical Skorokhod problems and their applications to queueing theory, risk theory and financial mathematics, is an actively investigated field of applied probability. In this paper we analyze $0-1$ properties of a class of such processes, that due to its importance in queueing theory (and dual risk theory) gained substantial interest; see, e.g., Norros (2004), Piterbarg (2001), Asmussen (2003), and Asmussen and Albrecher (2010) or novel works on $\gamma$-reflected Gaussian processes (Hashorva et al. 2013; Liu et al. 2015).

Consider a reflected (at 0) fractional Brownian motion with drift $Q_{B_{H}}=$ $\left\{Q_{B_{H}}(t): t \geq 0\right\}$, given by the following formula

$$
Q_{B_{H}}(t)=B_{H}(t)-c t+\max \left(Q_{B_{H}}(0),-\inf _{s \in[0, t]}\left(B_{H}(s)-c s\right)\right),
$$

where $c>0$ and $B_{H}=\left\{B_{H}(t): t \in \mathbb{R}\right\}$ is a fractional Brownian motion (fBm) with Hurst parameter $H \in(0,1)$, i.e., a centered Gaussian process with covariance function $\operatorname{Cov}\left(B_{H}(t), B_{H}(s)\right)=\frac{1}{2}\left(|t|^{2 H}+|s|^{2 H}-|t-s|^{2 H}\right)$. We focus on the investigation of the long-time behavior of the unique stationary solution of (1), which has the following representation

$$
Q_{B_{H}}(t)=\sup _{-\infty<s \leq t}\left(B_{H}(t)-B_{H}(s)-c(t-s)\right) .
$$

With no loss of generality in the reminder of this paper we assume that the drift parameter $c \equiv 1$. An important stimulus to analyze the distributional properties of $Q_{B_{H}}$ and its functionals stems from the Gaussian fluid queueing theory, where the stationary buffer content process in a queue which is fed by $B_{H}$ and emptied with constant rate $c=1$ is described by (2); see e.g. Norros (2004). In particular, in the seminal paper by Hüsler and Piterbarg (1999) the exact asymptotics of one dimensional marginal distributions of $Q_{B_{H}}$ was derived; see also Dieker (2005), Dębicki (2002), and Dębicki and Liu (2016) for results on more general Gaussian input processes.

The purpose of this paper is to investigate the asymptotic $0-1$ behavior of the processes $Q_{B_{H}}$. Our first contribution is an analog of the classical finding of Watanabe (1970), where an asymptotic 0-1 type of behavior for centered stationary Gaussian processes was analyzed.

Theorem 1. For all functions $f(t)$ that are positive and nondecreasing on some interval $[T, \infty)$, it follows that

$$
\mathbb{P}\left(Q_{B_{H}}(t)>f(t) \quad \text { i.o. }\right)=0 \text { or } 1,
$$

according as the integral

$$
\mathscr{I}_{f}:=\int_{T}^{\infty} \frac{1}{f(u)} \mathbb{P}\left(\sup _{t \in[0, f(u)]} Q_{B_{H}}(t)>f(u)\right) \mathrm{d} u
$$

is finite or infinite. 
The exact asymptotics, as $u$ grows large, of the probability in $\mathscr{I}_{f}$ was found by Piterbarg (2001, Theorem 7). Namely, for any $T>0$,

$\mathbb{P}\left(\sup _{t \in[0, T f(u)]} Q_{B_{H}}(t)>f(u)\right)=\sqrt{\pi} a^{\frac{2}{H}} b^{-\frac{1}{2}} \mathcal{H}_{B_{H}}^{2} T\left(v_{f}(u)\right)^{\frac{2}{H}-1} \Psi\left(v_{f}(u)\right)(1+o(1))$, as $u \rightarrow \infty$,

where $v_{f}(u)=A f^{1-H}(u), \Psi(u)=1-\Phi(u), \Phi$ is the distribution function of the unit normal law and the constants $a, b, A, \mathcal{H}_{B_{H}}$ are given explicitly in Section 2. Since relation (3) also holds when $T=T(u) \rightarrow 0$, provided that $T(u)(f(u))^{(1-H) / H} \rightarrow \infty$, we have that for $H \in\left(0, \frac{1}{2}\right)$, as $u \rightarrow \infty$,

$$
\frac{1}{f(u)} \mathbb{P}\left(\sup _{t \in[0, f(u)]} Q_{B_{H}}(t)>f(u)\right) \sim \mathbb{P}\left(\sup _{t \in[0,1]} Q_{B_{H}}(t)>f(u)\right) .
$$

Theorem 1 provides a tractable criterion for settling the dichotomy of $\mathbb{P}\left(Q_{B_{H}}(t)>f(t)\right.$ i.o. $)$. For instance, let $C_{H}=\left(2(1-H)^{2}-H\right) /(2 H(1-H))$ and

$$
f_{p}(s)=\left(\frac{2}{A^{2}}\left(\log s+\left(1+C_{H}-p\right) \log _{2} s\right)\right)^{\frac{1}{2(1-H)}}, \quad p \in \mathbb{R}, H \in(0,1) .
$$

One can check that, as $u \rightarrow \infty$,

$$
\frac{1}{f_{p}(u)} \mathbb{P}\left(\sup _{t \in\left[0, f_{p}(u)\right]} Q_{B_{H}}(t)>f_{p}(u)\right)=\frac{a^{\frac{2}{H}} b^{-\frac{1}{2}}}{\sqrt{2}} \mathcal{H}_{B_{H}}^{2} A^{\frac{1}{1-H}} 2^{C_{H}}\left(u \log ^{1-p} u\right)^{-1}(1+o(1)) .
$$

Hence, for any $p \in \mathbb{R}$,

$$
\mathbb{P}\left(Q_{B_{H}}(t)>f_{p}(t) \quad \text { i.o. }\right)=\left\{\begin{array}{l}
1 \text { if } p \geq 0 \\
0 \text { if } p<0
\end{array}\right.
$$

Corollary 1. For any $H \in(0,1)$,

$$
\limsup _{t \rightarrow \infty} \frac{Q_{B_{H}}(t)}{(\log t)^{\frac{1}{2(1-H)}}}=\left(\frac{2}{A^{2}}\right)^{\frac{1}{2(1-H)}} \text { a.s. }
$$

This result extends findings of Zeevi and Glynn (2000, Theorem 1), where it was proven that the above convergence holds weakly as well as in $L_{p}$ for all $p \in[1, \infty)$.

Now consider the process $\xi_{p}=\left\{\xi_{p}(t): t \geq 0\right\}$ defined as

$$
\xi_{p}(t)=\sup \left\{s: 0 \leq s \leq t, Q_{B_{H}}(s) \geq f_{p}(s)\right\} .
$$

Since $\mathscr{I}_{f_{p}}=\infty$ for $p \geq 0$, from Theorem 1 it follows that

$$
\lim _{t \rightarrow \infty} \xi_{p}(t)=\infty \quad \text { a.s. } \quad \text { and } \quad \limsup _{t \rightarrow \infty}\left(\xi_{p}(t)-t\right)=0 \quad \text { a.s. }
$$


Let, cf. (5),

$$
h_{p}(t)=p\left(\frac{1}{f_{p}(t)} \mathbb{P}\left(\sup _{s \in\left[0, f_{p}(t)\right]} Q_{B_{H}}(s)>f_{p}(t)\right)\right)^{-1} \log _{2} t .
$$

The second contribution of this paper is an Erdös-Révész type of law of the iterated logarithm for the process $\xi_{p}$. We refer to Shao (1992) for more background and references on Erdös-Révész type law of the iterated logarithm and a related result for centered stationary Gaussian processes; see also Dębicki and Kosiński (2016) for extensions to order statistics.

Theorem 2. If $p>1$, then

$$
\liminf _{t \rightarrow \infty} \frac{\xi_{p}(t)-t}{h_{p}(t)}=-1 \text { a.s. }
$$

If $p \in(0,1]$, then

$$
\liminf _{t \rightarrow \infty} \frac{\log \left(\xi_{p}(t) / t\right)}{h_{p}(t) / t}=-1 \text { a.s. }
$$

Now, let us complementary put $\eta_{p}=\left\{\eta_{p}(t): t \geq 0\right\}$, where

$$
\eta_{p}(t)=\inf \left\{s \geq t: Q_{B_{H}}(s) \geq f_{p}(s)\right\} .
$$

Since

$$
\mathbb{P}\left(\xi_{p}(t)-t \leq-x\right)=\mathbb{P}\left(\sup _{s \in(t-x, t]} \frac{Q_{B_{H}}(s)}{f_{p}(s)}<1\right)
$$

and

$$
\mathbb{P}\left(z-\eta_{p}(z) \leq-x\right)=\mathbb{P}\left(\sup _{s \in[z, z+x]} \frac{Q_{B_{H}}(s)}{f_{p}(s)}<1\right),
$$

then it follows that

$$
\liminf _{t \rightarrow \infty} \frac{\xi_{p}(t)-t}{h_{p}(t)}=\liminf _{z \rightarrow \infty} \frac{z-\eta_{p}(z)}{h_{p}(z)} .
$$

Theorem 2 shows that for $t$ big enough, there exists an $s$ in $\left[t-h_{p}(t), t\right]$ (as well as in $\left[t, t+h_{p}(t)\right]$ by (6)) such that $Q_{B_{H}}(s) \geq f_{p}(s)$ and that the length $h_{p}(t)$ of the interval is the smallest possible. This shines new light on results, which are intrinsically connected with Gumbel limit theorems; see, e.g., Leadbetter et al. (1983), where the function $h_{p}(t)$ plays crucial role. We shall pursue this elsewhere.

The paper is organized as follows. In Section 2 we introduce some useful properties of storage processes fed by fractional Brownian motion. In Section 3 we provide a collection of basic results on how to interpret extremes of the storage process $Q_{B_{H}}$ as extremes of a Gaussian field related to the fractional Brownian motion $B_{H}$. Furthermore, in Section 4 we prove lemmas, which constitute building blocks of the proofs of the main results. 


\section{Properties of the storage process}

In this section we introduce some notation and state some properties of the supremum of the process $Q_{B_{H}}$ as derived in Piterbarg (2001) and Hüsler and Piterbarg (2004b). We begin with the relation

$$
\mathbb{P}\left(\sup _{t \in[0, T]} Q_{B_{H}}(t)>u\right)=\mathbb{P}\left(\sup _{\substack{s \in[0, T / u] \\ \tau \geq 0}} Z_{u}(s, \tau)>u^{1-H}\right), \quad \text { for any } T>0,
$$

where, with $v(\tau)=\tau^{-H}+\tau^{1-H}$,

$$
Z_{u}(s, \tau):=\frac{B_{H}(u(\tau+s))-B_{H}(s u)}{\tau^{H} u^{H} v(\tau)}
$$

is a Gaussian field. Note that the self-similarity property of $B_{H}$ implies that the field $Z_{u}$ has the same distribution for any $u$. Thus, we do not use $u$ as an additional parameter in the following notation whenever it is not needed; let $Z(s, \tau):=$ $Z_{1}(s, \tau)$. Furthermore, the field $Z(s, \tau)$ is stationary in $s$, but not in $\tau$. The variance $\sigma_{Z}^{2}(\tau)$ of the field $Z(s, \tau)$ equals $\nu^{-2}(\tau)$ and $\sigma_{Z}(\tau)$ has a single maximum point at

$$
\tau_{0}=\frac{H}{1-H}
$$

Taylor expansion leads to

$$
\sigma_{Z}(\tau)=\frac{1}{A}-\frac{B}{2 A^{2}}\left(\tau-\tau_{0}\right)^{2}+O\left(\left(\tau-\tau_{0}\right)^{3}\right),
$$

as $\tau \rightarrow \tau_{0}$, where

$$
\begin{aligned}
& A=\frac{1}{1-H}\left(\frac{H}{1-H}\right)^{-H}=v\left(\tau_{0}\right), \\
& B=H\left(\frac{H}{1-H}\right)^{-H-2}=v^{\prime \prime}\left(\tau_{0}\right) .
\end{aligned}
$$

Let us define the correlation function of the process $Z_{u}$ as follows

$$
\begin{aligned}
& r_{u, u^{\prime}}\left(s, \tau, s^{\prime}, \tau^{\prime}\right):=\mathbb{E} Z_{u}(s, \tau) Z_{u}\left(s^{\prime}, \tau^{\prime}\right) v(\tau) v\left(\tau^{\prime}\right) \\
& =\frac{\left|u s-u^{\prime} s^{\prime}\right|^{2 H}}{2\left(u \tau u^{\prime} \tau^{\prime}\right)^{H}}\left(\left|1+\frac{u \tau}{\left(u s-u^{\prime} s^{\prime}\right)}\right|^{2 H}-\left|1+\frac{\left(u \tau-u^{\prime} \tau^{\prime}\right)}{\left(u s-u^{\prime} s^{\prime}\right)}\right|^{2 H}+\left|1-\frac{u^{\prime} \tau^{\prime}}{\left(u s-u^{\prime} s^{\prime}\right)}\right|^{2 H}-1\right) .
\end{aligned}
$$

By series expansion we find for any fixed $\tau_{1}<\tau_{0}<\tau_{2}$ and $\tau$, $\tau^{\prime}$ with $0<\tau_{1}<$ $\tau, \tau^{\prime}<\tau_{2}<\infty$

$$
\left|r_{u, u^{\prime}}\left(s, \tau, s^{\prime}, \tau^{\prime}\right)\right| \leq \frac{\left|u s-u^{\prime} s^{\prime}\right|^{2 H}}{\left(u \tau u^{\prime} \tau^{\prime}\right)^{H}} 2 H|2 H-1|\left|u s-u^{\prime} s^{\prime}\right|^{-2}\left(u \tau u^{\prime} \tau^{\prime}\right), \quad 2 H \neq 1,
$$


provided that $\left|\frac{u}{u s-u^{\prime} s^{\prime}}\right|$ and $\left|\frac{u^{\prime}}{u s-u^{\prime} s^{\prime}}\right|$ are sufficiently small. For $2 H=1$, we have $r_{u, u^{\prime}}\left(s, \tau, s^{\prime}, \tau^{\prime}\right)=0$ since the increments of Brownian motion on disjoint intervals are independent. Therefore,

$$
r^{*}(t):=\sup _{\substack{\frac{u s-u^{\prime} s^{\prime}}{u}|,| \frac{u s-u^{\prime} s^{\prime}}{u^{\prime}} \mid \geq t \\ \tau_{1}<\tau, \tau^{\prime}<\tau_{2} \\ u, u^{\prime}, s, s^{\prime}>0}}\left|r_{u, u^{\prime}}\left(s, \tau, s^{\prime}, \tau^{\prime}\right)\right| \leq \mathcal{K} t^{-\lambda},
$$

for $\lambda=2-2 H>0, t$ sufficiently large and some positive constant $\mathcal{K}$ depending only on $H, \tau_{1}$ and $\tau_{2}$. Similarly, from (8) it follows that for any fixed $M$ there exists $\delta \in(0,1)$ such that

$$
\begin{aligned}
0<\delta & \leq \inf _{\substack{\frac{u s-u^{\prime} s^{\prime}}{u}|,| \frac{u s-u^{\prime} s^{\prime}}{u^{\prime}}|\leq M\\
| \tau-\tau^{*}|,| \tau^{\prime}-\tau^{*} \mid \leq m}} r_{u, u^{\prime}}\left(s, \tau, s^{\prime}, \tau^{\prime}\right) \\
& \leq \sup _{\substack{\left|\frac{u s-u^{\prime} s^{\prime}}{u}\right|,|| \tau-\tau^{*}|,| \tau^{\prime}-\tau^{*} \mid \leq m}} r_{u, u^{\prime}}\left(s, \tau, s^{\prime}, \tau^{\prime}\right) \leq 1-\delta<1,
\end{aligned}
$$

for sufficiently small $m$.

\subsection{Asymptotics}

Due to the following lemma, while analyzing tail asymptotics of the supremum of $Z$, we can restrict the considered domain of $(s, \tau)$ to a strip with $\left|\tau-\tau_{0}\right| \leq \log v / v$.

Lemma 1 (Piterbarg (2001, Lemma 2 and 4)) There exists a positive constant $C$ such that for any $v, T>0$,

$$
\mathbb{P}\left(\sup _{\substack{s \in[0, T] \\\left|\tau-\tau_{0}\right| \geq \log v / v}} A Z(s, \tau)>v\right) \leq C T v^{2 / H} \exp \left(-\frac{1}{2} v^{2}-b \log ^{2} v\right),
$$

where $b=B /(2 A)$. Furthermore, for any $T>0$, with $a=1 /\left(2 \tau_{0}^{2 H}\right)$, as $v \rightarrow \infty$,

$$
\mathbb{P}\left(\sup _{\substack{s \in[0, T] \\\left|\tau-\tau_{0}\right| \leq \log v / v}} A Z(s, \tau)>v\right)=\sqrt{\pi} a^{\frac{2}{H}} b^{-\frac{1}{2}} \mathcal{H}_{B_{H}}^{2} T v^{\frac{2}{H}-1} \Psi(v)(1+o(1)),
$$

where

$$
\mathcal{H}_{B_{H}}=\lim _{T \rightarrow \infty} T^{-1} \mathbb{E} \exp \left(\sup _{t \in[0, T]}\left(\sqrt{2} B_{H}(t)-t^{2 H}\right)\right) \in(0, \infty),
$$

is the so-called Pickands' constant. This holds also for $T=v^{-1 / H^{\prime}}$, with $1>H^{\prime}>$ $H$. 
Hüsler and Piterbarg (2004a, Corollary 2) showed that the above actually holds true for $T$ depending on $v$ such that $v^{-1 / H^{\prime}}<T<\exp \left(c v^{2}\right)$, for any $H^{\prime} \in(H, 1)$ and $c \in\left(0, \frac{1}{2}\right)$.

\subsection{Discretization}

Let $\tau^{*}(v)=\log v / v$ and $J(v)=\left\{\tau:\left|\tau-\tau_{0}\right| \leq \tau^{*}(v)\right\}$. For a fixed $T, \theta>0$ and some $v>0$, let us define a discretization of the set $[0, T] \times J(v)$ as follows

$$
\begin{aligned}
s_{l} & =l q(v), \quad 0 \leq l \leq L, \quad L=[T / q(v)], \quad q(v)=\theta v^{-\frac{1}{H}}, \\
\tau_{n} & =\tau_{0}+n q(v), \quad 0 \leq|n| \leq N, \quad N=\left[\tau^{*}(v) / q(v)\right] .
\end{aligned}
$$

Along the same lines as in Hüsler and Piterbarg (2004b, Lemma 6) we get the following lemma.

Lemma 2 There exist positive constants $K_{1}, K_{2}, v_{0}>0$, such that, for any $\theta>0$ and $v \geq v_{0}$,

$\mathbb{P}\left(\max _{\substack{0 \leq l \leq L \\ 0 \leq|n| \leq N}} A Z\left(s_{l}, \tau_{n}\right) \leq v-\frac{\theta^{\frac{H}{2}}}{v}, \sup _{\substack{s \in[0, T] \\ \tau \in J(v)}} A Z(s, \tau)>v\right) \leq K_{1} v^{\frac{2}{H}-1} \Psi(v) \theta^{\frac{H}{2}} \exp \left(-\theta^{-H} / K_{2}\right)$.

Finally, it is possible to approximate tail asymptotics of the supremum of $Z$ on the strip $[0, T] \times J(v)$ by maximum taken over discrete time points. The proof of the following lemma follows line-by-line the same as the proof of Piterbarg (2001, Lemma 4) and thus we omit it. Similar result can be found in, e.g., Hüsler and Piterbarg (2004b, Lemma 7).

Lemma 3 For any $T, \theta>0$, as $v \rightarrow \infty$,

$$
\mathbb{P}\left(\max _{\substack{0 \leq l \leq L \\ 0 \leq|n| \leq N}} A Z\left(s_{l}, \tau_{n}\right)>v\right)=\sqrt{\pi} a^{\frac{2}{H}} b^{-\frac{1}{2}}\left(\mathcal{H}_{B_{H}}^{\theta}\right)^{2} T v^{\frac{2}{H}-1} \Psi(v)(1+o(1)),
$$

where $\mathcal{H}_{B_{H}}^{\theta}=\lim _{S \rightarrow \infty} S^{-1} \mathbb{E} \exp \left(\sup _{t \in \theta \mathbb{Z} \cap[0, S]}\left(\sqrt{2} B_{H}(t)-t^{2 H}\right)\right)$.

It follows easily that $\mathcal{H}_{B_{H}}^{\theta} \rightarrow \mathcal{H}_{B_{H}}$ as $\theta \rightarrow 0$, so that the above asymptotics is the same as in Lemma 1 when the discretization parameter $\theta$ decreases to zero so that the number of discretization points grows to infinity.

\section{Auxiliary lemmas}

We begin with some auxiliary lemmas that are later needed in the proofs. The first lemma is a slightly modified version of Leadbetter et al. (1983, Theorem 4.2.1). 
Lemma 4 (Berman's inequality) Suppose that $\xi_{1}, \ldots, \xi_{n}$ are normal random variables with correlation matrix $\Lambda^{1}=\left(\Lambda_{i, j}^{1}\right)$ and $\eta_{1}, \ldots, \eta_{n}$ similarly with correlation matrix $\Lambda^{0}=\left(\Lambda_{i, j}^{0}\right)$. Let $\sigma\left(\xi_{i}\right)=\sigma\left(\eta_{i}\right) \in(0,1], \rho_{i, j}=\max \left(\left|\Lambda_{i, j}^{1}\right|,\left|\Lambda_{i, j}^{0}\right|\right)$ and $u_{i}$ be real numbers, $i=1, \ldots, n$. Then,

$$
\begin{aligned}
\mathbb{P}\left(\bigcap_{j=1}^{n}\left\{\xi_{j} \leq u_{j}\right\}\right) & -\mathbb{P}\left(\bigcap_{j=1}^{n}\left\{\eta_{j} \leq u_{j}\right\}\right) \\
& \leq \frac{1}{2 \pi} \sum_{1 \leq i<j \leq n}\left(\Lambda_{i, j}^{1}-\Lambda_{i, j}^{0}\right)^{+}\left(1-\rho_{i, j}^{2}\right)^{-\frac{1}{2}} \exp \left(-\frac{u_{i}^{2}+u_{j}^{2}}{2\left(1+\rho_{i, j}\right)}\right)
\end{aligned}
$$

The following lemma is a general form of the Borel-Cantelli lemma; cf. (Spitzer 1964).

Lemma 5 (Borel-Cantelli lemma) Consider a sequence of events $\left\{E_{k}\right\}_{k=0}^{\infty}$. If

$$
\sum_{k=0}^{\infty} \mathbb{P}\left(E_{k}\right)<\infty
$$

then $\mathbb{P}\left(E_{n}\right.$ i.o. $)=0$. Whereas, if

$$
\sum_{k=0}^{\infty} \mathbb{P}\left(E_{k}\right)=\infty \quad \text { and } \quad \liminf _{n \rightarrow \infty} \frac{\sum_{1 \leq k \neq t \leq n} \mathbb{P}\left(E_{k} E_{t}\right)}{\left(\sum_{k=1}^{n} \mathbb{P}\left(E_{k}\right)\right)^{2}} \leq 1,
$$

then $\mathbb{P}\left(E_{n}\right.$ i.o. $)=1$.

Lemma 6 For any $\varepsilon \in(0,1)$, there exist positive constants $K$ and $\rho$ depending only on $\varepsilon, H, p$ and $\lambda$ such that

$$
\begin{aligned}
\mathbb{P}\left(\sup _{S<t \leq T} \frac{Q_{B_{H}}(t)}{f_{p}(t)} \leq 1\right) \leq & \exp \left(-\frac{(1-\varepsilon)}{(1+\varepsilon)} \int_{S+f_{p}(S)}^{T} \frac{1}{f_{p}(u)} \mathbb{P}\left(\sup _{t \in\left[0, f_{p}(u)\right]} Q_{B_{H}}(t)>f_{p}(u)\right) \mathrm{d} u\right) \\
& +K S^{-\rho},
\end{aligned}
$$

for any $T-f_{p}(S) \geq S \geq K$, with $f_{p}(T) / f_{p}(S) \leq \mathcal{C}$ and $\mathcal{C}$ being some universal positive constant.

Proof Let $\varepsilon \in(0,1)$ be some positive constant. For the reminder of the proof let $K$ and $\rho$ be two positive constants depending only on $\varepsilon, H, p$ and $\lambda$ that may differ from line to line. For any $k \geq 0$ put $s_{0}=S, y_{0}=f_{p}\left(s_{0}\right), t_{0}=s_{0}+y_{0}, x_{0}=f_{p}\left(t_{0}\right)$ and

$$
\begin{aligned}
& s_{k}=t_{k-1}+\varepsilon x_{k-1}, \quad y_{k}=f_{p}\left(s_{k}\right), \quad t_{k}=s_{k}+y_{k}, \quad x_{k}=f_{p}\left(t_{k}\right), \\
& I_{k}=\left(s_{k}, t_{k}\right], \quad v_{k}=A x_{k}^{1-H}=v_{f_{p}}\left(t_{k}\right), \quad \tilde{I}_{k}=\frac{I_{k}}{x_{k}}=\left(\tilde{s}_{k}, \tilde{t}_{k}\right], \quad\left|\tilde{I}_{k}\right|=\frac{y_{k}}{x_{k}} .
\end{aligned}
$$

From this construction, it is easy to see that the intervals $I_{k}$ are disjoint. Furthermore, $\delta\left(I_{k}, I_{k+1}\right)=\varepsilon x_{k}$, and $1-\varepsilon \leq y_{k} / x_{k} \leq 1$, for any $k \geq 0$ and sufficiently large $S$. 
Note that, for any $k \geq 0,\left|I_{k}\right| \sim f_{p}(S)$ as $S$ grows large, therefore if $T(S, \varepsilon)$ is the smallest number of intervals $\left\{I_{k}\right\}$ needed to cover $[S, T]$, then $T(S, \varepsilon) \leq[(T-$ $\left.S) /\left(f_{p}(S)(1+\varepsilon)\right)\right]$. Moreover, since $f_{p}(T) / f_{p}(S)$ is bounded by the constant $\mathcal{C}>0$ not depending on $S$ and $\varepsilon$, it follows that, $x_{k} / x_{t} \leq \mathcal{C}$ for any $0 \leq t<k \leq T(S, \varepsilon)$.

Now let us introduce a discretization of the set $\tilde{I}_{k} \times J\left(v_{k}\right)$ as in Section 2.2. That is, for some $\theta>0$, define grid points

$$
\begin{aligned}
s_{k, l} & =\tilde{s}_{k}+l q_{k}, \quad 0 \leq l \leq L_{k}, \quad L_{k}=\left[(1-\varepsilon) / q_{k}\right], \quad q_{k}=\theta v_{k}^{-\frac{1}{H}}, \\
\tau_{k, n} & =\tau_{0}+n q_{k}, \quad 0 \leq|n| \leq N_{k}, \quad N_{k}=\left[\tau^{*}\left(v_{k}\right) / q_{k}\right] .
\end{aligned}
$$

Since $f_{p}$ is an increasing function, it easily follows that,

$$
\begin{aligned}
& \mathbb{P}\left(\sup _{S<t \leq T} \frac{Q_{B_{H}}(t)}{f_{p}(t)} \leq 1\right) \leq \mathbb{P}\left(\bigcap_{k=0}^{T(S, \varepsilon)}\left\{\sup _{t \in I_{k}} Q_{B_{H}}(t) \leq x_{k}\right\}\right) \\
& \leq \mathbb{P}\left(\bigcap_{k=0}^{T(S, \varepsilon)}\left\{\sup _{\substack{s \in I_{k} / x_{k} \\
\tau \in J\left(v_{k}\right)}} A Z_{x_{k}}(s, \tau) \leq v_{k}\right\}\right) \\
& \leq \mathbb{P}\left(\bigcap_{k=0}^{T(S, \varepsilon)}\left\{\max _{\substack{0 \leq l \leq L_{k} \\
0 \leq|n| \leq N_{k}}} A Z_{x_{k}}\left(s_{k, l}, \tau_{k, n}\right) \leq v_{k}\right\}\right) \\
& \leq \prod_{k=0}^{T(S, \varepsilon)} \mathbb{P}\left(\max _{\substack{0 \leq l \leq L_{k} \\
0 \leq|n| \leq N_{k}}} A Z_{x_{k}}\left(s_{k, l}, \tau_{k, n}\right) \leq v_{k}\right) \\
& +\sum_{0 \leq t<k \leq T(S, \varepsilon)} C_{k, t}=: P_{1}+P_{2}
\end{aligned}
$$

where the last inequality follows from Berman's inequality with

$$
C_{k, t}=\sum_{\substack{0 \leq l \leq L_{k} \\ 0 \leq p \leq L_{t}}} \sum_{\substack{|n| \leq N_{k} \\|m| \leq N_{t}}} \frac{\left|r_{x_{k}, x_{t}}\left(s_{k, l}, \tau_{k, n}, s_{t, p}, \tau_{t, m}\right)\right|}{\sqrt{1-r_{x_{k}, x_{t}}^{2}\left(s_{k, l}, \tau_{k, n}, s_{t, p}, \tau_{t, m}\right)}} \exp \left(-\frac{\frac{1}{2}\left(v_{k}^{2}+v_{t}^{2}\right)}{1+\left|r_{x_{k}, x_{t}}\left(s_{k, l}, \tau_{k, n}, s_{t, p}, \tau_{t, m}\right)\right|}\right)
$$

\section{Estimate of $P_{1}$.}

Note that we can use the fact that $Z_{x_{k}}$ has the same distribution as $Z_{1} \equiv Z$ for any $x_{k}$. Since the process $Z$ is stationary with respect to the first variable, from Lemma 3 , for any $\varepsilon \in(0,1)$, sufficiently large $S$ and small $\theta$,

$$
\begin{aligned}
P_{1} & \leq \exp \left(-\sum_{k=0}^{T(S, \varepsilon)} \mathbb{P}\left(\max _{\substack{0 \leq l \leq L_{k} \\
0 \leq|n| \leq N_{k}}} A Z_{x_{k}}\left(s_{k, l}, \tau_{k, n}\right)>v_{k}\right)\right) \\
& \leq \exp \left(-\left(1-\frac{\varepsilon}{4}\right) \sum_{k=0}^{T(S, \varepsilon)} \mathbb{P}\left(\sup _{(s, \tau) \in \tilde{I}_{k} \times J\left(v_{k}\right)} A Z(s, \tau)>v_{k}\right)\right)
\end{aligned}
$$


Then, by (7) combined with (3),

$$
\begin{aligned}
P_{1} & \leq \exp \left(-\left(1-\frac{\varepsilon}{2}\right) \sum_{k=0}^{T(S, \varepsilon)} \mathbb{P}\left(\sup _{\substack{\tilde{I}_{k} \\
\tau \geq 0}} A Z(s, \tau)>v_{k}\right)\right) \\
& =\exp \left(-\left(1-\frac{\varepsilon}{2}\right) \sum_{k=0}^{T(S, \varepsilon)} \mathbb{P}\left(\sup _{t \in\left[0, \frac{y_{k}}{x_{k}} f_{p}\left(t_{k}\right)\right]} Q_{B_{H}}(t)>f_{p}\left(t_{k}\right)\right)\right) \\
& \leq \exp \left(-(1-\varepsilon) \sum_{k=0}^{T(S, \varepsilon)} \mathbb{P}\left(\sup _{t \in\left[0, f_{p}\left(t_{k}\right)\right]} Q_{B_{H}}(t)>f_{p}\left(t_{k}\right)\right) \frac{f_{p}\left(s_{k}\right)}{f_{p}\left(t_{k}\right)}\right) \\
& \leq \exp \left(-\frac{1-\varepsilon}{1+\varepsilon} \int_{S+f_{p}(S)}^{T} \frac{1}{f_{p}(u)} \mathbb{P}\left(\sup _{t \in\left[0, f_{p}(u)\right]} Q_{B_{H}}(t)>f_{p}(u)\right) \mathrm{d} u\right) .
\end{aligned}
$$

\section{Estimate of $P_{2}$.}

For any $0 \leq t<k \leq T(S, \varepsilon), 0 \leq l \leq L_{k}, 0 \leq p \leq L_{t}$, we have

$$
\begin{aligned}
x_{k} s_{k, l}-x_{t} s_{t, p} & =\left(s_{k}+x_{k} l q_{k}\right)-\left(s_{t}+x_{t} p q_{t}\right) \\
& =\sum_{i=t}^{k-1}\left(y_{i}+\varepsilon x_{i}\right)+x_{k} l q_{k}-x_{t} p q_{t} \geq \sum_{i=t}^{k-1}\left(y_{i}+\varepsilon x_{i}\right)-x_{t}(1-\varepsilon) \\
& \geq\left(y_{t}+\varepsilon x_{t}\right)(k-t)-x_{t}(1-\varepsilon) \geq x_{t}(k-t) \varepsilon
\end{aligned}
$$

where the last inequality holds provided that $k-t \geq s_{0}$ with $s_{0}$ sufficiently large. Therefore, c.f. (9),

$$
\begin{aligned}
r_{k, t}^{*} & :=\sup _{\substack{0 \leq l \leq L_{k}, 0 \leq p \leq L_{t} \\
|n| \leq N_{k},|m| \leq N_{t}}}\left|r_{x_{k}, x_{t}}\left(s_{k, l}, \tau_{k, n}, s_{t, p}, \tau_{t, m}\right)\right| \leq r^{*}((k-t) \varepsilon) \leq \mathcal{K}(k-t)^{-\lambda} \\
& \leq \min (1, \lambda) / 4 .
\end{aligned}
$$

Moreover, from (10) it follows that, for any $0 \leq k-t \leq s_{0}$, there exists a constant $\zeta \in(0,1)$ depending only on $\varepsilon$ such that for sufficiently large $S$,

$$
\sup _{\substack{0 \leq l \leq L_{k}, 0 \leq p \leq L_{t} \\|n| \leq N_{k},|m| \leq N_{t}}}\left|r_{x_{k}, x_{t}}\left(s_{k, l}, \tau_{k, n}, s_{t, p}, \tau_{t, m}\right)\right| \leq \zeta<1 .
$$


Finally, recall that $N_{k} \leq L_{k} \leq \theta^{-1} v_{k}^{\frac{1}{H}}$ and $\exp \left(-v_{k}^{2} / 2\right)=\left(t_{k} \log ^{\left(1+C_{H}-p\right)} t_{k}\right)^{-1}$, c.f. (4),(12), so that

$$
\begin{aligned}
& P_{2} \leq \frac{4}{\sqrt{1-\zeta^{2}}} \sum_{0 \leq t<k \leq T(S, \varepsilon)} L_{k} L_{t} N_{k} N_{t} r_{k, t}^{*} \exp \left(-\frac{v_{k}^{2}+v_{t}^{2}}{2\left(1+r_{k, t}^{*}\right)}\right) \\
& \leq K\left(\sum_{\substack{0<k-t \leq s_{0} \\
0 \leq t<k \leq T(S, \varepsilon)}}+\sum_{\substack{k-t>s_{0} \\
0 \leq t<k \leq T(S, \varepsilon)}}\right)(\cdot) \\
& \leq K\left(\sum_{k=0}^{\infty} v_{k}^{\frac{4}{H}} \exp \left(-\frac{v_{k}^{2}}{1+\zeta}\right)+\sum_{\substack{k-t>s_{0} \\
0 \leq t<k \leq T(S, \varepsilon)}} v_{k}^{\frac{2}{H}} v_{t}^{\frac{2}{H}}(k-t)^{-\lambda} \exp \left(-\frac{v_{k}^{2}+v_{t}^{2}}{2\left(1+\frac{\lambda}{4}\right)}\right)\right) \\
& \leq K\left(\sum_{k=0}^{\infty} t_{k}^{-\frac{2}{1+\sqrt{\zeta}}}+\sum_{\substack{k-t>s_{0} \\
0 \leq t<k \leq T(S, \varepsilon)}} t_{k}^{-\frac{1}{1+\frac{\lambda}{2}}} t_{t}^{-\frac{1}{1+\frac{\lambda}{2}}}(k-t)^{-\lambda}\right) \\
& \leq K\left(\sum_{k=[S]}^{\infty} k^{-\frac{2}{1+\sqrt{\zeta}}}+\sum_{[S] \leq t<k \leq \infty} k^{-\frac{1}{1+\frac{\lambda}{2}}} t^{-\frac{1}{1+\frac{\lambda}{2}}}(k-t)^{-\lambda}\right) \\
& \leq K S^{-\rho} \text {, }
\end{aligned}
$$

where the last inequality follows from basic algebra.

Let $S>0$ be any fixed number, $a_{0}=S, y_{0}=f_{p}\left(a_{0}\right)$ and $b_{0}=a_{0}+y_{0}$. For $i>0$, define

$$
\begin{aligned}
a_{i} & =b_{i-1}, \quad y_{i}=f_{p}\left(a_{i}\right), \quad b_{i}=a_{i}+y_{i}, \quad M_{i}=\left(a_{i}, b_{i}\right], \quad v_{i}=A y_{i}^{1-H}, \\
\tilde{M}_{i} & =\frac{M_{i}}{y_{i}}=\left(\tilde{a}_{i}, \tilde{b}_{i}\right] .
\end{aligned}
$$

From this construction it is easy to see that the intervals $M_{i}$ are disjoint, $\cup_{j=0}^{i} M_{j}=$ $\left(S, b_{i}\right]$ and $\left|\tilde{M}_{i}\right|=1$. Now let us introduce a discretization of the set $\tilde{M}_{i} \times J\left(v_{i}\right)$ as in Section 2.2. That is, for some $\theta>0$, define grid points

$$
\begin{aligned}
s_{i, l} & =\tilde{a}_{i}+l q_{i}, \quad 0 \leq l \leq L_{i}, \quad L_{i}=\left[1 / q_{i}\right], \quad q_{i}=\theta v_{i}^{-\frac{1}{H}}, \\
\tau_{i, n} & =\tau_{0}+n q_{i}, \quad 0 \leq|n| \leq N_{i}, \quad N_{i}=\left[\tau^{*}\left(v_{i}\right) / q_{i}\right] .
\end{aligned}
$$

With the above notation, we have the following lemma. 
Lemma 7 For any $\varepsilon \in(0,1)$ there exist positive constants $K$ and $\rho$ depending only on $\varepsilon, H, p$ and $\lambda$ such that, with $\theta_{i}=v_{i}^{-4 / H}$,

$$
\begin{aligned}
& \mathbb{P}\left(\bigcap_{i=0}^{\left[(T-S) / f_{p}(S)\right]}\left\{\max _{\substack{0 \leq l \leq L_{i} \\
0 \leq|n| \leq N_{i}}} A Z_{y_{i}}\left(s_{i, l}, \tau_{i, n}\right) \leq v_{i}-\frac{\theta_{i}^{\frac{H}{2}}}{v_{i}}\right\}\right) \\
& \geq \frac{1}{4} \exp \left(-(1+\varepsilon) \int_{S}^{T} \frac{1}{f_{p}(u)} \mathbb{P}\left(\sup _{t \in\left[0, f_{p}(u)\right]} Q_{B_{H}}(t)>f_{p}(u)\right) \mathrm{d} u\right)-K S^{-\rho},
\end{aligned}
$$

for any $T-f_{p}(S) \geq S \geq K$, with $f_{p}(T) / f_{p}(S) \leq \mathcal{C}$ and $\mathcal{C}$ being some universal positive constant.

Proof Put $\hat{v}_{i}=v_{i}-\theta_{i}^{\frac{H}{2}} / v_{i}$ and $I=\left[(T-S) / f_{p}(S)\right]$. Similarly as in the proof of Lemma 6 we find that Berman's inequality implies

$$
\begin{aligned}
& \mathbb{P}\left(\bigcap_{i=0}^{I}\left\{\max _{\substack{0 \leq l \leq L_{i} \\
0 \leq|n| \leq N_{i}}} A Z_{y_{i}}\left(s_{i, l}, \tau_{i, n}\right) \leq v_{i}-\frac{\theta_{i}^{\frac{H}{2}}}{v_{i}}\right\}\right) \\
& \geq \prod_{i=0}^{I} \mathbb{P}\left(\max _{\substack{0 \leq l \leq L_{i} \\
0 \leq|n| \leq N_{i}}} A Z_{y_{i}}\left(s_{i, l}, \tau_{i, n}\right) \leq v_{i}-\frac{\theta_{i}^{\frac{H}{2}}}{v_{i}}\right)-\sum_{0 \leq i<j \leq I} D_{i, j}=: P_{1}^{\prime}+P_{2}^{\prime},
\end{aligned}
$$

where

$$
\begin{aligned}
D_{i, j}= & \frac{1}{2 \pi} \sum_{\substack{0 \leq l \leq L_{i} \\
0 \leq p \leq L_{j}}} \sum_{\substack{|n| \leq N_{i} \\
|m| \leq N_{j}}} \frac{\left(\tilde{r}_{y_{i}, y_{j}}\left(s_{i, l}, \tau_{i, n}, s_{j, p}, \tau_{j, m}\right)\right)^{+}}{\sqrt{1-\tilde{r}_{y_{i}, y_{j}}^{2}\left(s_{i, l}, \tau_{i, n}, s_{j, p}, \tau_{j, m}\right)}} \\
& \times \exp \left(-\frac{\frac{1}{2}\left(\hat{v}_{i}^{2}+\hat{v}_{j}^{2}\right)}{1+\left|\tilde{r}_{y_{i}, y_{j}}\left(s_{i, l}, \tau_{i, n}, s_{j, p}, \tau_{j, m}\right)\right|}\right),
\end{aligned}
$$

with

$$
\tilde{r}_{y_{i}, y_{j}}\left(s_{i, l}, \tau_{i, n}, s_{j, p}, \tau_{j, m}\right)=-r_{y_{i}, y_{j}}\left(s_{i, l}, \tau_{i, n}, s_{j, p}, \tau_{j, m}\right) .
$$

Estimate of $P_{1}^{\prime}$.

By Lemma 1 the correction term $\theta_{i}^{\frac{H}{2}} / v_{i}$ does not change the order of the asymptotics of the tail of $Z$. Furthermore, the tail asymptotics of the supremum on the strip 
$(s, \tau) \in \tilde{M}_{i} \times J\left(v_{i}\right)$ are of the same order if $\tau \geq 0$. Hence, for every $\varepsilon>0$, following the same lines of reasoning as in the estimation of $P_{1}$ in Lemma 6 ,

$$
\begin{aligned}
& P_{1}^{\prime} \geq \prod_{i=0}^{I}\left(1-\mathbb{P}\left(\max _{\substack{0 \leq l \leq L_{i} \\
0 \leq|n| \leq N_{i}}} A Z_{y_{i}}\left(s_{i, l}, \tau_{i, n}\right)>\hat{v}_{i}\right)\right) \\
& \geq \frac{1}{4} \exp \left(-\sum_{i=0}^{I} \mathbb{P}\left(\max _{\substack{0 \leq l \leq L_{i} \\
0 \leq|n| \leq N_{i}}} A Z_{y_{i}}\left(s_{i, l}, \tau_{i, n}\right)>\hat{v}_{i}\right)\right) \\
& \geq \frac{1}{4} \exp \left(-\sum_{i=0}^{I} \mathbb{P}\left(\sup _{\substack{s \in \tilde{M}_{i} \\
\tau \in J\left(v_{i}\right)}} A Z(s, \tau)>v_{i}-\frac{\theta_{i}^{\frac{H}{2}}}{v_{i}}\right)\right) \\
& \geq \frac{1}{4} \exp \left(-(1+\varepsilon) \sum_{i=0}^{I} \mathbb{P}\left(\sup _{\substack{s \in \tilde{M}_{i} \\
\tau \geq 0}} A Z(s, \tau)>v_{i}\right)\right) \\
& =\frac{1}{4} \exp \left(-(1+\varepsilon) \sum_{i=0}^{I} \mathbb{P}\left(\sup _{t \in\left[0, f_{p}\left(a_{i}\right)\right]} Q_{B_{H}}(t)>f_{p}\left(a_{i}\right)\right)\right) \\
& \geq \frac{1}{4} \exp \left(-(1+\varepsilon) \int_{S}^{T} \frac{1}{f_{p}(u)} \mathbb{P}\left(\sup _{t \in\left[0, f_{p}(u)\right]} Q_{B_{H}}(t)>f_{p}(u)\right) \mathrm{d} u\right),
\end{aligned}
$$

provided that $S$ is sufficiently large.

\section{Estimate of $P_{2}^{\prime}$.}

Clearly, for $j \geq i+2$ and any $0 \leq l \leq L_{i}, 0 \leq p \leq L_{j}$; c.f. (13),

$$
y_{j} s_{j, p}-y_{i} s_{i, l}=a_{j}+y_{j} p q_{j}-\left(a_{i}+y_{i} l q_{i}\right) \geq(j-i-1) y_{i},
$$

so that by (9), for any $0 \leq i<j \leq I$,

$$
r_{i, j}^{*}:=\sup _{\substack{0 \leq l \leq L_{i}, 0 \leq p \leq L_{j} \\|n| \leq N_{i},|m| \leq N_{j}}}\left|\tilde{r}_{y_{i}, y_{j}}\left(s_{i, l}, \tau_{i, n}, s_{j, p}, \tau_{j, m}\right)\right| \leq r^{*}(j-i-1) \leq r^{*}(1)<1 .
$$

On the other hand, by (10), there exist positive constants $s_{0}$, such that for sufficiently large $S$,

$$
\begin{array}{ll}
\left(\tilde{r}_{y_{i}, y_{j}}\left(s_{i, l}, \tau_{i, n}, s_{j, p}, \tau_{j, m}\right)\right)^{+}=0, & \text { if } \quad j=i+1, \quad\left|y_{j} s_{j, p}-y_{i} s_{i, l}\right| / y_{i} \leq s_{0}, \\
\left|\tilde{r}_{y_{i}, y_{j}}\left(s_{i, l}, \tau_{i, n}, s_{j, p}, \tau_{j, m}\right)\right| \leq r^{*}\left(s_{0}\right)<1, & \text { if } \quad j=i+1, \quad\left|y_{j} s_{j, p}-y_{i} s_{i, l}\right| / y_{j}>s_{0}
\end{array}
$$


Therefore, by (15)-(16) we obtain

$$
\begin{aligned}
P_{2}^{\prime} \leq & \sum_{\substack{0 \leq i \leq I-1 \\
j=i+1}} \sum_{\substack{0 \leq l \leq L_{i} \\
0 \leq p \leq L_{j}}} \sum_{\substack{|n| \leq N_{i} \\
|m| \leq N_{j}}} \frac{1}{\sqrt{1-r^{*}\left(s_{0}\right)}} \exp \left(-\frac{\frac{1}{2}\left(\hat{v_{i}^{2}}+\hat{v_{j}^{2}}\right)}{1+r^{*}\left(s_{0}\right)}\right) \\
& +\sum_{\substack{0 \leq i \leq I-2 \\
i+2 \leq j \leq I \leq 1}} \sum_{\substack{0 \leq l \leq L_{i} \\
0 \leq p \leq L_{j}}} \sum_{\substack{|n| \leq N_{i} \\
|m| \leq N_{j}}} \frac{r^{*}(j-i-1)}{\sqrt{1-r^{*}(1)}} \exp \left(-\frac{\frac{1}{2}\left(\hat{v_{i}^{2}}+\hat{v}_{j}^{2}\right)}{1+r^{*}(j-i-1)}\right) .
\end{aligned}
$$

Completely similarly to the estimation of $P_{2}$ in the proof of Lemma 6, we can get that there exist positive constants $K$ and $\rho$ such that, for sufficiently large $S$,

$$
P_{2}^{\prime} \leq K S^{-\rho} \text {. }
$$

The next lemma is a straightforward modification of Watanabe (1970, Lemma 3.1 and Lemma 4.1), see also Qualls and Watanabe (1971, Lemma 1.4).

Lemma 8 If Theorem 1 is true under the additional condition, that for large $t$,

$$
\left(\frac{2}{A^{2}} \log t\right)^{1 / 2(1-H)} \leq f(t) \leq\left(\frac{3}{A^{2}} \log t\right)^{1 / 2(1-H)},
$$

it is true without the additional condition.

\section{Proof of the main results}

Proof of Theorem 1 Note that the case $\mathscr{I}_{f}<\infty$ is straightforward and does not need any additional knowledge on the process $Q_{B_{H}}$ apart from the stationarity property. Indeed, consider the sequence of intervals $M_{i}$ as in Lemma 7. Then, for any $\varepsilon>0$ and sufficiently large $T$,

$$
\sum_{k=[T]+1}^{\infty} \mathbb{P}\left(\sup _{t \in M_{k}} Q_{B_{H}}(t)>f\left(a_{k}\right)\right)=\sum_{k=[T]}^{\infty} \mathbb{P}\left(\sup _{t \in\left[0, f\left(b_{k}\right)\right]} Q_{B_{H}}(t)>f\left(b_{k}\right)\right) \leq \mathscr{I}_{f}<\infty,
$$

and the Borel-Cantelli lemma completes this part of the proof since $f$ is an increasing function.

Now let $f$ be an increasing function such that $\mathscr{I}_{f} \equiv \infty$. Using the same notation as in Lemma 6 with $f$ instead of $f_{p}$, we find that, for any $S, \varepsilon, \theta>0$,

$$
\begin{aligned}
\mathbb{P}\left(Q_{B_{H}}(s)>f(s) \text { i.o. }\right) & \geq \mathbb{P}\left(\left\{\sup _{t \in I_{k}} Q_{B_{H}}(t)>f\left(t_{k}\right)\right\} \text { i.o. }\right) \\
& \geq \mathbb{P}\left(\left\{\max _{\substack{0 \leq l \leq L_{k} \\
0 \leq|n| \leq N_{k}}} A Z_{x_{k}}\left(s_{k, l}, \tau_{k, n}\right)>v_{k}\right\} \text { i.o. }\right) .
\end{aligned}
$$


Let

$$
E_{k}=\left\{\max _{\substack{0 \leq l \leq L_{k} \\ 0 \leq|n| \leq N_{k}}} A Z_{x_{k}}\left(s_{k, l}, \tau_{k, n}\right) \leq v_{k}\right\}
$$

For sufficiently large $S$ and $\theta$; c.f. estimation of $P_{1}$, we get

$$
\sum_{k=0}^{\infty} \mathbb{P}\left(E_{k}^{c}\right) \geq \frac{(1-\varepsilon)}{(1+\varepsilon)} \int_{S+f(S)}^{\infty} \frac{1}{f(u)} \mathbb{P}\left(\sup _{t \in[0, f(u)]} Q_{B_{H}}(t)>f(u)\right) \mathrm{d} u=\infty .
$$

Note that

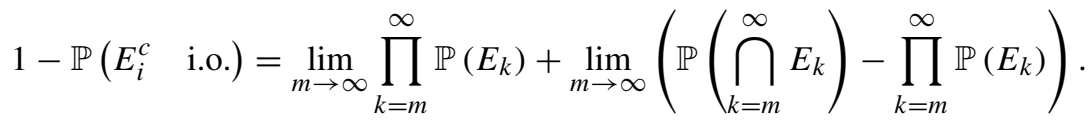

The first limit equals to zero as a consequence of (19). The second limit equals to zero because of the asymptotic independence of the events $E_{k}$. Indeed, there exist positive constants $K$ and $\rho$, depending only on $H, \varepsilon, \lambda$, such that for any $n>m$,

$$
A_{m, n}=\left|\mathbb{P}\left(\bigcap_{k=m}^{n} E_{k}\right)-\prod_{k=m}^{n} \mathbb{P}\left(E_{k}\right)\right| \leq K(S+m)^{-\rho},
$$

by the same calculations as in the estimate of $P_{2}$ in Lemma 6 after realizing that, by Lemma 8 , we might restrict ourselves to the case when (18) holds. Therefore $\mathbb{P}\left(E_{i}^{c}\right.$ i.o. $)=1$, which completes the proof.

Proof of Theorem 2 In order to make the proof more transparent we divide it on several steps.

Step 1. Let $p>1$. Then, for every $\varepsilon \in\left(0, \frac{1}{4}\right)$,

$$
\liminf _{t \rightarrow \infty} \frac{\xi_{p}(t)-t}{h_{p}(t)} \geq-(1+2 \varepsilon)^{2} \quad \text { a.s. }
$$

Proof Let $\left\{T_{k}: k \geq 1\right\}$ be a sequence such that $T_{k} \rightarrow \infty$, as $k \rightarrow \infty$. Put $S_{k}=$ $T_{k}-(1+2 \varepsilon)^{2} h_{p}\left(T_{k}\right)$. Since $h_{p}(t)=O\left(t \log ^{1-p} t \log _{2} t\right)$, then, for $p>1, S_{k} \sim T_{k}$, as $k \rightarrow \infty$, and from Lemma 6 it follows that

$$
\begin{aligned}
& \mathbb{P}\left(\frac{\xi_{p}\left(T_{k}\right)-T_{k}}{h_{p}\left(T_{k}\right)} \leq-(1+2 \varepsilon)^{2}\right)=\mathbb{P}\left(\xi_{p}\left(T_{k}\right) \leq S_{k}\right)=\mathbb{P}\left(\sup _{S_{k}<t \leq T_{k}} \frac{Q_{B_{H}}(t)}{f_{p}(t)}<1\right) \\
\leq & \exp \left(-\frac{(1-\varepsilon)}{(1+\varepsilon)} \int_{S_{k}+f_{p}\left(S_{k}\right)}^{T_{k}} \frac{1}{f_{p}(u)} \mathbb{P}\left(\sup _{t \in\left[0, f_{p}(u)\right]} Q_{B_{H}}(t)>f_{p}(u)\right) \mathrm{d} u\right)+2 K T_{k}^{-\rho} .
\end{aligned}
$$


Moreover, as $k \rightarrow \infty$,

$$
\begin{aligned}
& \int_{S_{k}+f_{p}\left(S_{k}\right)}^{T_{k}} \frac{1}{f_{p}(u)} \mathbb{P}\left(\sup _{t \in\left[0, f_{p}(u)\right]} Q_{B_{H}}(t)>f_{p}(u)\right) \mathrm{d} u \\
\sim & (1+2 \varepsilon)^{2} h_{p}\left(T_{k}\right) \frac{1}{f_{p}\left(T_{k}\right)} \mathbb{P}\left(\sup _{t \in\left[0, f_{p}\left(T_{k}\right)\right]} Q_{B_{H}}(t)>f_{p}\left(T_{k}\right)\right) \\
= & (1+2 \varepsilon)^{2} p \log _{2} T_{k} .
\end{aligned}
$$

Now take $T_{k}=\exp \left(k^{1 / p}\right)$. Then,

$$
\sum_{k=0}^{\infty} \mathbb{P}\left(\xi_{p}\left(T_{k}\right) \leq S_{k}\right) \leq 2 K \sum_{k=0}^{\infty} k^{-(1+\varepsilon / 2)}<\infty .
$$

Hence, by the Borel-Cantelli lemma, we have

$$
\liminf _{k \rightarrow \infty} \frac{\xi_{p}\left(T_{k}\right)-T_{k}}{h_{p}\left(T_{k}\right)} \geq-(1+2 \varepsilon)^{2} \quad \text { a.s.. }
$$

Since $\xi_{p}(t)$ is a non-decreasing random function of $t$, for every $T_{k} \leq t \leq T_{k+1}$, we have

$$
\frac{\xi_{p}(t)-t}{h_{p}(t)} \geq \frac{\xi_{p}\left(T_{k}\right)-T_{k}}{h_{p}\left(T_{k}\right)}-\frac{T_{k+1}-T_{k}}{h_{p}\left(T_{k}\right)} .
$$

For $p>1$ elementary calculus implies

$$
\lim _{k \rightarrow \infty} \frac{T_{k+1}-T_{k}}{h_{p}\left(T_{k}\right)}=0
$$

so that

$$
\liminf _{t \rightarrow \infty} \frac{\xi_{p}(t)-t}{h_{p}(t)} \geq \liminf _{k \rightarrow \infty} \frac{\xi_{p}\left(T_{k}\right)-T_{k}}{h_{p}\left(T_{k}\right)} \quad \text { a.s. },
$$

which completes the proof of this step.

Step 2. Let $p>1$. Then, for every $\varepsilon \in(0,1)$,

$$
\liminf _{t \rightarrow \infty} \frac{\xi_{p}(t)-t}{h_{p}(t)} \leq-(1-\varepsilon) \quad \text { a.s. }
$$

Proof As in the proof of the lower bound (Step 1), we put

$$
T_{k}=\exp \left(k^{\left(1+\varepsilon^{2}\right) / p}\right), \quad S_{k}=T_{k}-(1-\varepsilon) h_{p}\left(T_{k}\right), \quad k \geq 1 .
$$

Let

$$
B_{k}=\left\{\xi_{p}\left(T_{k}\right) \leq S_{k}\right\}=\left\{\sup _{S_{k}<t \leq T_{k}} \frac{Q_{B_{H}}(t)}{f_{p}(t)}<1\right\} .
$$


It suffices to show $\mathbb{P}\left(B_{n}\right.$ i.o. $)=1$, that is

$$
\lim _{m \rightarrow \infty} \mathbb{P}\left(\bigcup_{k=m}^{\infty} B_{k}\right)=1
$$

Let

$$
\begin{gathered}
a_{0}^{k}=S_{k}, \quad y_{0}^{k}=f_{p}\left(a_{0}^{k}\right), \quad b_{0}^{k}=a_{0}^{k}+y_{0}^{k}, \\
a_{i}^{k}=b_{i-1}^{k}, \quad y_{i}^{k}=f_{p}\left(a_{i}^{k}\right), \quad b_{i}^{k}=a_{i}^{k}+y_{i}^{k}, \quad M_{i}^{k}=\left(a_{i}^{k}, b_{i}^{k}\right], \quad v_{i}^{k}=A\left(y_{i}^{k}\right)^{1-H}, \\
\tilde{M_{i}^{k}}=\frac{M_{i}^{k}}{y_{i}^{k}}=\left(\tilde{a_{i}^{k}}, \tilde{b_{i}^{k}}\right] .
\end{gathered}
$$

Define $J_{k}$ to be the biggest number such that $b_{J_{k}-1}^{k} \leq T_{k}$ and $b_{J_{k}}^{k}>T_{k}$. In what follows let $b_{J_{k}}^{k}$ be redefined to $T_{k}$. Note that $J_{k} \leq\left[\left(T_{k}-S_{k}\right) / f_{p}\left(S_{k}\right)\right]$.

Since $f_{p}$ is an increasing function,

$$
\begin{aligned}
B_{k} & =\bigcap_{i=0}^{J_{k}}\left\{\sup _{t \in M_{i}^{k}} \frac{Q_{B_{H}}(t)}{f_{p}(t)}<1\right\} \supset \bigcap_{i=0}^{J_{k}}\left\{\sup _{t \in M_{i}^{k}} Q_{B_{H}}(t)<y_{i}^{k}\right\} \\
& =\bigcap_{i=0}^{J_{k}}\left\{\sup _{\substack{s \in \tilde{M}_{i}^{k} \\
\tau \geq 0}} A Z_{y_{i}^{k}}(s, \tau)<v_{i}^{k}\right\} .
\end{aligned}
$$

Analogously to (14), define a discretization of the set $\tilde{M}_{i}^{k} \times J\left(v_{i}^{k}\right)$ as follows

$$
\begin{aligned}
s_{i, l}^{k}=\tilde{a}_{i}^{k}+l q_{i}^{k}, & 0 \leq l \leq L_{i}^{k}, \quad L_{i}^{k}=\left[1 / q_{i}^{k}\right], \quad q_{i}^{k}=\theta_{i}^{k}\left(v_{i}^{k}\right)^{-\frac{1}{H}}, \quad \theta_{i}^{k}=\left(v_{i}^{k}\right)^{-\frac{4}{H}} \\
\tau_{i, n}^{k}=\tau_{0}+n q_{i}^{k}, & 0 \leq|n| \leq N_{i}^{k}, \quad N_{i}^{k}=\left[\tau^{*}\left(v_{i}^{k}\right) / q_{i}^{k}\right] .
\end{aligned}
$$

Finally, let

$$
A_{k}=\bigcap_{i=0}^{J_{k}}\left\{\max _{\substack{0 \leq l \leq L_{i}^{k} \\ 0 \leq|n| \leq N_{i}^{k}}} A Z_{y_{i}^{k}}\left(s_{i, l}^{k}, \tau_{i, n}^{k}\right) \leq v_{i}^{k}-\frac{\left(\theta_{i}^{k}\right)^{\frac{H}{2}}}{v_{i}^{k}}\right\}
$$

Observe that

$$
\mathbb{P}\left(\bigcup_{k=m}^{\infty} A_{k}\right) \leq \mathbb{P}\left(\bigcup_{k=m}^{\infty} B_{k}\right)+\sum_{k=m}^{\infty} \mathbb{P}\left(A_{k} \cap B_{k}^{c}\right)
$$


Furthermore,

$$
\begin{aligned}
\sum_{k=m}^{\infty} \mathbb{P}\left(A_{k} \cap B_{k}^{c}\right) \leq & \sum_{k=m}^{\infty} \sum_{i=0}^{J_{k}} \mathbb{P}\left(\max _{\substack{0 \leq l \leq L_{i}^{k} \\
0 \leq|n| \leq N_{i}^{k}}} A Z_{y_{i}^{k}}\left(s_{i, l}^{k}, \tau_{i, n}^{k}\right) \leq v_{i}^{k}-\frac{\left(\theta_{i}^{k}\right)^{\frac{H}{2}}}{v_{i}^{k}}, \sup _{\substack{s \in \tilde{M}_{i}^{k} \\
\tau \geq 0}} A Z_{y_{i}^{k}}(s, \tau) \geq v_{i}^{k}\right) \\
\leq & \sum_{k=m}^{\infty} \sum_{i=0}^{J_{k}} \mathbb{P}\left(\max _{\substack{0 \leq l \leq L_{i}^{k} \\
0 \leq|n| \leq N_{i}^{k}}} A Z_{y_{i}^{k}}\left(s_{i, l}^{k}, \tau_{i, n}^{k}\right) \leq v_{i}^{k}-\frac{\left(\theta_{i}^{k}\right)^{\frac{H}{2}}}{v_{i}^{k}}, \sup _{\substack{s \in \tilde{M}_{i}^{k} \\
\tau \in J\left(v_{i}^{k}\right)}} A Z_{y_{i}^{k}}(s, \tau) \geq v_{i}^{k}\right) \\
& +\sum_{k=m}^{\infty} \sum_{i=0}^{J_{k}} \mathbb{P}\left(\sup _{\substack{s \in \tilde{M}_{i}^{k} \\
\tau \notin J\left(v_{i}^{k}\right)}} A Z_{y_{i}^{k}}(s, \tau) \geq v_{i}^{k}\right) .
\end{aligned}
$$

By Lemma 2, for sufficiently large $m$ and some $K_{1}, K_{2}>0$, the first sum is bounded from above by

$$
\begin{aligned}
& K \sum_{k=m}^{\infty} \sum_{i=0}^{J_{k}}\left(v_{i}^{k}\right)^{\frac{2(1-2 H)}{H}} \exp \left(-\frac{\left(v_{i}^{k}\right)^{2}}{2}-\frac{\left(v_{i}^{k}\right)^{4}}{K_{1}}\right) \\
\leq & K \sum_{k=m}^{\infty} \sum_{i=0}^{J_{k}} \frac{\left(\log a_{i}^{k}\right)^{\frac{1-2 H}{H}}}{a_{i}^{k}\left(\log a_{i}^{k}\right)^{1+C_{H}-p}} \exp \left(-\frac{\log ^{2}\left(a_{i}^{k}\right)}{K_{2}}\right) \\
\leq & K \sum_{k=m}^{\infty} \sum_{i=0}^{J_{k}}\left(S_{k}+i f_{p}\left(S_{k}\right)\right)^{-2} \leq K \sum_{k=m}^{\infty}\left(S_{k}\right)^{-1} \leq K m^{-4} .
\end{aligned}
$$

Note that by (11), for sufficiently large $m$, the term in (23) is bounded from above by

$$
\begin{aligned}
& K \sum_{k=m}^{\infty} \sum_{i=0}^{J_{k}}\left(v_{i}^{k}\right)^{\frac{2}{H}} \exp \left(-\frac{1}{2}\left(v_{i}^{k}\right)^{2}-b \log ^{2} v_{i}^{k}\right) \\
\leq & K \sum_{k=m}^{\infty} \sum_{i=0}^{J_{k}} \frac{1}{a_{i}^{k} \log ^{1+p} a_{i}^{k}} \leq K \sum_{k=m}^{\infty} \sum_{i=S_{k}}^{\infty} \frac{1}{i \log ^{1+p} i} \\
\leq & K \sum_{k=m}^{\infty}\left(\log \left(S_{k}\right)\right)^{-p} \leq K \sum_{k=m}^{\infty} k^{-(1+\varepsilon)^{2}} \leq K m^{-\varepsilon} .
\end{aligned}
$$

Therefore

$$
\lim _{m \rightarrow \infty} \sum_{k=m}^{\infty} \mathbb{P}\left(A_{k} \cap B_{k}^{c}\right)=0
$$

and

$$
\lim _{m \rightarrow \infty} \mathbb{P}\left(\bigcup_{k=m}^{\infty} B_{k}\right) \geq \lim _{m \rightarrow \infty} \mathbb{P}\left(\bigcup_{k=m}^{\infty} A_{k}\right)
$$


In order to complete the proof of (22) we only need to show that

$$
\mathbb{P}\left(A_{n} \text { i.o. }\right)=1 \text {. }
$$

Similarly to (20), we have

$$
\int_{S_{k}}^{T_{k}} \frac{1}{f_{p}(u)} \mathbb{P}\left(\sup _{t \in\left[0, f_{p}(u)\right]} Q_{B_{H}}(t)>f_{p}(u)\right) \mathrm{d} u \sim(1-\varepsilon) p \log _{2} T_{k} .
$$

Now from Lemma 7 it follows that

$$
\mathbb{P}\left(A_{k}\right) \geq \frac{1}{4} \exp \left(-\left(1-\varepsilon^{2}\right) p \log _{2} T_{k}\right)-K S_{k}^{-\rho} \geq \frac{1}{8} k^{-\left(1-\varepsilon^{4}\right)},
$$

for every $k$ sufficiently large. Hence,

$$
\sum_{k=1}^{\infty} \mathbb{P}\left(A_{k}\right)=\infty
$$

Applying Berman's inequality, we get for $t<k$

$$
\mathbb{P}\left(A_{k} A_{t}\right) \leq \mathbb{P}\left(A_{k}\right) \mathbb{P}\left(A_{t}\right)+Q_{k, t},
$$

where

$$
\begin{aligned}
Q_{k, t}= & \sum_{\substack{0 \leq i \leq J_{k} \\
0 \leq j \leq J_{t}}} \sum_{\substack{0 \leq l \leq L_{i}^{k} \\
0 \leq p \leq L_{j}^{t}|n| \leq N_{i}^{k}|m| \leq N_{j}^{t}}} \frac{\left|r_{y_{i}^{k}, y_{j}^{t}}\left(s_{i, l}^{k}, \tau_{i, n}^{k}, s_{j, p}^{t}, \tau_{j, m}^{t}\right)\right|}{\sqrt{1-r_{y_{i}^{k}, y_{j}^{t}}^{2}\left(s_{i, l}^{k}, \tau_{i, n}^{k}, s_{j, p}^{t}, \tau_{j, m}^{t}\right)}} \\
& \times \exp \left(-\frac{\left(v_{i}^{k}-\left(v_{i}^{k}\right)^{-3}\right)^{2}+\left(v_{j}^{t}-\left(v_{j}^{t}\right)^{-3}\right)^{2}}{2\left(1+\left|r_{y_{i}^{k}, y_{j}^{t}}\left(s_{i, l}^{k}, \tau_{i, n}^{k}, s_{j, p}^{t}, \tau_{j, m}^{t}\right)\right|\right)}\right) .
\end{aligned}
$$

For any $0 \leq i \leq J_{k}, 0 \leq j \leq J_{t}, 0 \leq l \leq L_{i}^{k}, 0 \leq p \leq L_{j}^{t}$, and $t<k$, $y_{i}^{k} s_{i, l}^{k}-y_{j}^{t} s_{j, p}^{t}=a_{i}^{k}+y_{i}^{k} l q_{i}^{k}-\left(a_{j}^{t}+y_{j}^{t} p q_{j}^{t}\right) \geq S_{k}-T_{t} \geq S_{k}-T_{k-1} \geq \frac{1}{2}\left(T_{k}-T_{k-1}\right)$, where the last inequality holds for $k$ large enough, since

$$
\frac{S_{k+1}-T_{k}}{T_{k+1}-T_{k}} \sim 1, \quad \text { as } k \rightarrow \infty .
$$

Thus, for sufficiently large $k$ and every $0 \leq t<k$, c.f. (9),

$$
\begin{aligned}
& \sup _{\substack{0 \leq i \leq J_{k} \\
0 \leq j \leq J_{t} \\
0 \leq l \leq L_{i}^{k}, 0 \leq p \leq L_{j}^{t} \\
|n| \leq N_{i}^{k},|m| \leq N_{j}^{t}}}\left|r_{y_{i}^{k}, y_{j}^{t}}\left(s_{i, l}^{k}, \tau_{n}^{k}, s_{j, p}^{t}, \tau_{j, m}^{t}\right)\right| \leq \sup _{0 \leq i \leq J_{k}} r^{* \leq j \leq J_{t}} \text { ( }\left(\frac{T_{k}-T_{k-1}}{2 \sqrt{y_{i}^{k} y_{j}^{k}}}\right) \\
& \quad \leq \mathcal{K}\left(\frac{T_{k}-T_{k-1}}{2 f_{p}\left(T_{k}\right)}\right)^{-\lambda} \leq \mathcal{K}\left(T_{k}-T_{k-1}\right)^{-\lambda / 2} \leq \frac{\min (1, \lambda)}{32} .
\end{aligned}
$$


Therefore, for some generic constant $K$ not depending on $k$ and $t$ which may vary between lines, for every $t<k$ sufficiently large,

$$
\begin{aligned}
Q_{k, t} & \leq K \sum_{\substack{0 \leq i \leq J_{k} \\
0 \leq j \leq J_{t}}} L_{i}^{k} L_{j}^{t} N_{i}^{k} N_{j}^{t}\left(T_{k}-T_{k-1}\right)^{-\lambda / 2} \exp \left(-\frac{\left(v_{i}^{k}\right)^{2}+\left(v_{j}^{t}\right)^{2}}{2\left(1+\frac{\lambda}{16}\right)}\right) \\
& \leq K\left(T_{k}-T_{k-1}\right)^{-\lambda / 2}\left(L_{J_{k}}^{k} L_{J_{t}}^{t}\right)^{2} \sum_{\substack{0 \leq i \leq J_{k} \\
0 \leq j \leq J_{t}}}\left(a_{i}^{k} \log ^{1+C_{H}-p} a_{i}^{k}\right)^{-\frac{1}{1+\frac{\lambda}{16}}}\left(a_{j}^{t} \log ^{1+C_{H}-p} a_{j}^{t}\right)^{-\frac{1}{1+\frac{\lambda}{16}}} \\
& \leq K\left(T_{k}-T_{k-1}\right)^{-\lambda / 2} \log ^{10} T_{k}\left(T_{k}\right)^{\frac{\frac{\lambda}{8}}{1+\frac{\lambda}{8}}}\left(T_{t}\right)^{\frac{\frac{\lambda}{8}}{1+\frac{\lambda}{8}}} \\
& \leq K T_{k}^{-\lambda / 8} \leq K \exp \left(-\lambda k^{\left(1+\varepsilon^{2}\right) / p} / 8\right) .
\end{aligned}
$$

Hence, we have

$$
\sum_{0 \leq t<k<\infty} Q_{k, t}<\infty .
$$

Now (24) follows from (25)-(27) and the general form of the Borel-Cantelli lemma.

Step 3. If $p \in(0,1]$, then for every $\varepsilon \in\left(0, \frac{1}{4}\right)$

$$
\liminf _{t \rightarrow \infty} \frac{\log \left(\xi_{p}(t) / t\right)}{h_{p}(t) / t} \geq-(1+2 \varepsilon)^{2} \quad \text { a.s. }
$$

and

$$
\liminf _{t \rightarrow \infty} \frac{\log \left(\xi_{p}(t) / t\right)}{h_{p}(t) / t} \leq-(1-\varepsilon) \quad \text { a.s. }
$$

Proof Put

$$
T_{k}=\exp (k), \quad S_{k}=T_{k} \exp \left(-(1+2 \varepsilon)^{2} h_{p}\left(T_{k}\right)\right) .
$$

Proceeding the same as in the proof of (21), one can obtain that

$$
\liminf _{k \rightarrow \infty} \frac{\log \left(\xi_{p}\left(T_{k}\right) / T_{k}\right)}{h_{p}\left(T_{k}\right) / T_{k}} \geq-(1+2 \varepsilon)^{2} \quad \text { a.s. }
$$

On the other hand it is clear that

$$
\liminf _{t \rightarrow \infty} \frac{\log \left(\xi_{p}(t) / t\right)}{h_{p}(t) / t}=\liminf _{k \rightarrow \infty} \frac{\log \left(\xi_{p}\left(T_{k}\right) / T_{k}\right)}{h_{p}\left(T_{k}\right) / T_{k}} \quad \text { a.s. }
$$

since

This proves (28).

$$
\liminf _{k \rightarrow \infty} \frac{\log \left(T_{k} / T_{k+1}\right)}{h_{p}\left(T_{k}\right) / T_{k}}=0
$$

Let

$$
T_{k}=\exp \left(k^{1+\varepsilon^{2}}\right), \quad S_{k}=T_{k} \exp \left(-(1-\varepsilon) h_{p}\left(T_{k}\right)\right) .
$$

Noting that

$$
\frac{S_{k+1}-T_{k}}{S_{k+1}} \sim 1, \quad \text { as } k \rightarrow \infty,
$$


following along the same lines as in the proof of (22), we also have

$$
\liminf _{k \rightarrow \infty} \frac{\log \left(\xi_{p}\left(T_{k}\right) / T_{k}\right)}{h_{p}\left(T_{k}\right) / T_{k}} \leq-(1-\varepsilon) \quad \text { a.s. }
$$

which proves (29).

Acknowledgements We are thankful to the editor and the referee for several suggestions which improved our manuscript. K. Dębicki was partially supported by National Science Centre Grant No. 2015/17/B/ST1/01102 (2016-2019). Research of K. Kosiński was conducted under scientific Grant No. 2014/12/S/ST1/00491 funded by National Science Centre.

Open Access This article is distributed under the terms of the Creative Commons Attribution 4.0 International License (http://creativecommons.org/licenses/by/4.0/), which permits unrestricted use, distribution, and reproduction in any medium, provided you give appropriate credit to the original author(s) and the source, provide a link to the Creative Commons license, and indicate if changes were made.

\section{References}

Asmussen, S.: Applied Probability and Queues. Springer 2nd edn (2003)

Asmussen, S., Albrecher, H.: Ruin Probabilities. World Scientific Publishing Co. Inc., 2nd edn (2010)

Dieker, A.B.: Extremes of Gaussian processes over an infinite horizon. Stoch. Process. Appl. 115, 207-248 (2005)

Dębicki, K.: Ruin probability for Gaussian integrated processes. Stoch. Process. Appl. 98, 151-174 (2002)

Dębicki, K., Kosiński, K.M.: An Erdös-Révész type law of the iterated logarithm for order statistics of a stationary Gaussian process. J. Theor. Probab. doi:10.1007/s10959-016-0710-8 (2016)

Dębicki, K., Liu, P.: Extremes of stationary Gaussian storage models. Extremes 19(2), 273-302 (2016)

Hashorva, E., Ji, L., Piterbarg, V.I.: On the supremum of $\gamma$-reflected processes with fractional Brownian motion as input. Stoch. Process. Appl. 123, 4111-4127 (2013)

Hüsler, J., Piterbarg, V.I.: Extremes of a certain class of Gaussian processes. Stoch. Process. Appl. 83, 257-271 (1999)

Hüsler, J., Piterbarg, V.I.: On the ruin probability for physical fractional Brownian motion. Stoch. Process. Appl. 113, 315-332 (2004a)

Hüsler, J., Piterbarg, V.I.: Limit theorem for maximum of the storage process with fractional Brownian motion as input. Stoch. Process. Appl. 114, 231-250 (2004b)

Leadbetter, M.R., Lindgren, G., Rootzen, H.: Extremes and Related Properties of Random Sequences and Processes. Springer, Berlin Heidelberg New York (1983)

Liu, P., Hashorva, E., Ji, L.: On the $\gamma$-reflected processes with fBm input. Lith. Math. J 55(3), $402-412$ (2015)

Norros, I.: A storage model with self-similar input. Queueing Syst. 16, 387-396 (2004)

Piterbarg, V.I.: Large deviations of a storage process with fractional Brownian motion as input. Extremes 4, 147-164 (2001)

Qualls, C., Watanabe, H.: An asymptotic 0-1 behavior of Gaussian processes. Ann. Math. Stat. 42(6), 2029-2035 (1971)

Shao, Q.-M.: An Erdös-Révész type law of the iterated logarithm for stationary Gaussian processes. Probab. Theory Relat. Fields 94(1), 119-133 (1992)

Spitzer, F.: Principles of Random Walk. Van Nostrand, Princeton (1964)

Watanabe, H.: An asymptotic property of Gaussian processes. Amer. Math. Soc. 148(1), 233-248 (1970)

Zeevi, A.J., Glynn, P.W.: On the maximum workload of a queue fed by fractional Brownian motion. Ann. Appl. Probab. 10(4), 1084-1099 (2000) 